\title{
BMJ Open Varicella zoster virus-associated morbidity and mortality in Africa: a systematic review protocol
}

\author{
Hannah S Hussey, ${ }^{1}$ Leila H Abdullahi, ${ }^{2}$ Jamie E Collins, ${ }^{3}$ Rudzani Muloiwa, ${ }^{4}$ \\ Gregory D Hussey, ${ }^{2}$ Benjamin M Kagina ${ }^{2}$
}

To cite: Hussey HS, Abdullahi LH, Collins JE, et al. Varicella zoster virusassociated morbidity and mortality in Africa: a systematic review protocol. BMJ Open 2016;6:e010213. doi:10.1136/bmjopen-2015010213

- Prepublication history for this paper is available online. To view these files please visit the journal online (http://dx.doi.org/10.1136/ bmjopen-2015-010213)

Received 8 October 2015 Revised 6 February 2016 Accepted 8 February 2016

CrossMark

For numbered affiliations see end of article.

Correspondence to Dr Hannah S Hussey; hshussey@gmail.com

\section{ABSTRACT}

Introduction: Varicella zoster virus (VZV) causes varicella (chicken pox) and herpes zoster (shingles). Worldwide, these diseases are associated with significant morbidity. Most of the epidemiological data on VZV come from high income countries. There are few data on VZV in Africa, where tropical climates and high HIV/AIDS prevalence rates are expected to impact the epidemiology of VZV. Safe and effective vaccinations for both varicella and herpes zoster exist, but are not routinely used in Africa. There are very few data available on VZV disease burden in Africa to guide the introduction of these vaccines on the continent. Our aim is to conduct a systematic review of the VZV-associated morbidity and mortality in Africa, which will provide critical information that could be used to develop vaccination policies against these diseases in Africa.

Methods and analysis: Electronic databases will be searched and all studies published after 1974 that meet predefined criteria will be assessed. The primary outcomes for the study are VZV incidence/prevalence, hospitalisation rates and total death rates. The secondary outcome for this study is the proportion of VZV hospitalisations and/or deaths associated with HIV/ AIDS. Two reviewers will screen the titles and abstracts, and then independently review the full texts, to determine if studies are eligible for inclusion. A risk of bias and quality assessment tool will be used to score all included studies. Following standardised data extraction, a trend analysis using R-programming software will be conducted to investigate the trend of VZV. Depending on the characteristics of included studies, subgroup analyses will be performed. This review will be reported according to the Preferred Reporting Items for Systematic reviews and MetaAnalyses (PRISMA) guidelines.

Ethics and dissemination: As this is a protocol for a systematic review, which will use already published data, no ethics approval is required. Findings will be disseminated in peer-reviewed journals.

Trial registration number: CRD42015026144.

\section{BACKGROUND}

Varicella zoster virus (VZV), belonging to the Herpesviridae family, causes varicella

\section{Strengths and limitations of this study}

- This is the first time data on Varicella zoster virus (VZV) in Africa have been collated in a systematic review.

- The data reporting adheres to the Preferred Reporting Items for Systematic reviews and Meta-Analyses (PRISMA) guidelines.

- Publication bias - only published studies will be included in the review. Data from national health departments will not be used as there is no standardised data collection for VZV across the continent.

- If the majority of the data are collected from healthcare facilities, cases that do not have access to these facilities will be missed, making the study further underestimate the burden of VZV in Africa.

(chicken pox) and, later, through endogenous reactivation, herpes zoster (shingles). Both diseases occur worldwide, and while their mortality is generally low, these diseases have a high morbidity, and place a large burden on healthcare systems and society. ${ }^{1}$ There is no synthesised literature on the burden of varicella and herpes zoster in Africa.

Varicella is characterised by a vesicular rash and fever, and in children is usually a mild, self-limiting illness. Complications, however, do occur, particularly in infants, pregnant women and other adults, and in immunocompromised individuals, including those with HIV. ${ }^{1}{ }^{2}$ Globally, on average, 4.2 million cases of severe varicella result in hospitalisation or death each year. ${ }^{2}$ Prior to widespread usage of vaccines against varicella in temperate high income countries, 13-16 cases of varicella per 1000 population occurred annually, mostly affecting children aged $1-9$ years. ${ }^{1}$ In these settings, $>90 \%$ of the population becomes infected with VZV before adolescence. ${ }^{2}$ In tropical regions, primary infection of varicella tends to occur at a later stage, 
resulting in a larger population of susceptible adults and potentially a higher proportion of severe cases. ${ }^{23}$

After an episode of varicella, VZV remains dormant in the dorsal root ganglia, and reactivation results in zoster, a painful vesicular rash, usually confined to one dermatome. ${ }^{4}$ The incidence of herpes zoster increases with increasing age, especially after 50 years of life. For example, half of all 85-year-old individuals have experienced an episode of herpes zoster. ${ }^{2}$ Decreasing cellmediated immunity due to, for example, HIV infection, cancer, diabetes mellitus or immunosuppressive treatment, also increases the risk of zoster. ${ }^{2}$ Compared with HIV-negative individuals, persons with HIV have a 1217-fold greater risk of developing zoster. In areas with a high HIV prevalence, zoster has an $85-95 \%$ positive predictive value for underlying HIV infection. ${ }^{5}$ The most common complications of zoster are post-herpetic neuralgia and ocular complications. ${ }^{6}$ About $3 \%$ of zoster patients are hospitalised. ${ }^{7}$ There are few data on zoster mortality, but studies from Europe and North America suggest that it is around 0.25 per 1 million population, mostly in the elderly. ${ }^{7}$ Timely vaccination against zoster may prevent such deaths.

Effective vaccines exist for both varicella and zoster, and are used widely in high-income countries, with considerable benefits. ${ }^{2}$ In 1998, the WHO recommended the introduction of routine childhood vaccination against VZV in settings where the disease has significant negative socioeconomic impact. ${ }^{8}$ But vaccines against VZV are rarely used on the African continent. Introducing these vaccines would be in agreement with the Global Immunisation Vision and Strategy (GIVS) of the WHO and UNICEF. The GIVS aims to widely introduce a range of newly available vaccines and immunise more people against as many vaccine-preventable diseases as possible. ${ }^{9}$ Achieving this aim would promote health, but would also improve equitable access to immunisation. ${ }^{9}$

Decisions to introduce vaccines against varicella and zoster in most African countries need to be guided by evidence-based data on the epidemiology and socioeconomic impacts of the diseases. To the best of our knowledge, at present, there are no synthesised VZV-associated epidemiological data in Africa. Furthermore, cost-effectiveness studies of introducing the vaccines against varicella and shingles are lacking in Africa.

The African continent has several risk factors that could result in an increased burden of VZV disease. First, for varicella, there is a high prevalence of HIV/ AIDS and, in tropical countries, primary infections occur at an older age, both of which increase the chance of developing severe varicella. Second, for zoster, there is an increasing ageing population, high HIV/ AIDS burden and diabetes prevalence is growing. ${ }^{10} 11$ Third, for both varicella and zoster, weak and overstretched healthcare systems in Africa cannot efficiently manage complications of these diseases.
Rationale

Globally, major variations in the epidemiology of VZV-associated disease exist. As such, some countries have adopted universal childhood vaccination against VZV while others recommend targeted vaccination of high-risk populations only. ${ }^{1}$ In Africa, there is no collated evidence on the burden of varicella and zoster that could be used to inform decisions around vaccine introduction for these diseases. Furthermore, the impact of the HIV/AIDS pandemic on the epidemiology of VZV is also not clear, and any decisions around introduction of new vaccines on the continent would have to take this into account too.

\section{METHODS \\ Objective}

This study attempts to describe the epidemiology of VZV in Africa, taking into account the clinical diseases of varicella and zoster.

\section{Primary objectives}

- To summarise the available data on the varicellaassociated morbidity and mortality in Africa

- To summarise the available data on the herpes zosterassociated morbidity and mortality in Africa.

\section{Secondary objective}

To assess the impact of HIV/AIDS on the epidemiology of varicella and herpes zoster in Africa.

\section{Eligibility criteria}

Types of participants

Studies on the epidemiology of VZV, in adults and children, from any country in Africa, will be included in the review.

\section{Case definition}

Included studies must have clearly stated the case definition for varicella. The case definition for varicella, as defined by the Centres for Disease Control and Prevention (CDC), will be used for morbidity and mortality estimation: ${ }^{12}$

Clinical description: An acute illness with diffuse maculopapulovesicular rash, without other apparent cause.

Laboratory criteria: Isolation of varicella virus from a clinical specimen, or varicella antigen detected by direct fluorescent antibody test, or varicella-specific nucleic acid detected by PCR, or significant rise in serum antivaricella $\operatorname{IgG}$ antibody level by any standard serologic assay.

The case definition of herpes zoster uses only the clinical description (painful maculopapulovesicular rash, usually confined to a dermatome), as it has been shown to be distinctive enough to make an accurate clinical diagnosis. ${ }^{13}$ 


\section{Inclusion criteria}

Studies will be included if they are conducted in Africa, measure or report any of the primary outcomes listed below and have a case definition for varicella or herpes zoster.

\section{Exclusion criteria}

Studies will be excluded from this review if they are not conducted in Africa, do not measure any of the primary outcomes listed below and have not stated a case definition of varicella or herpes zoster.

\section{Outcomes}

\section{Primary outcomes}

- Incidence or prevalence of varicella or herpes zoster

- Hospitalisation rates associated with varicella or herpes zoster

- Total deaths associated with varicella or herpes zoster.

\section{Secondary outcomes}

Proportion of varicella or herpes zoster hospitalisations and/or deaths associated with HIV/AIDS.

\section{Type of studies}

Case-series, cross-sectional, cohort and intervention studies will be included. All studies published from January 1974 to September 2015 will be included without language restrictions. First, Google translator software will be used to enable preliminary screening of non-English records by titles or abstracts that appear likely to be included. If the article still appears likely for inclusion, we will then seek translation support from a native speaker of the language used in the article, within our network of collaborators. If unsuccessful with this option, we will seek professional translation services.

\section{Search strategy}

The literature search strategy will use both text words and Medical Subject Heading (MeSH) terms and will include the following: VZV, human herpes virus 3, varicella, chicken pox, herpes zoster and shingles, as well as prevalence, incidence, epidemiology, burden, hospitalisation or hospitalization, and mortality and case-fatality rates. These terms will be adapted for each database and then combined with a relevant filter to select out studies from the African continent only. An example of the PubMed search strategy is shown in table 1.

\section{Electronic databases}

The following electronic databases will be searched: PubMed, Scopus, Africa-wide, Embase, WHOLIS, PDQ-Evidence, CENTRAL, CINAHL and Web of Science.

\section{Selection of eligible studies}

The first and the second authors (HSH and LHA) will screen the search outputs using titles and abstracts. In addition, study setting, study design, methods as well as

\begin{tabular}{|c|c|}
\hline $\begin{array}{l}\text { Query } \\
\text { number }\end{array}$ & Search terms \\
\hline$\# 1$ & $\begin{array}{l}\text { 'Herpesvirus 3, Human'[Mesh] OR 'Varicella } \\
\text { zoster' }\end{array}$ \\
\hline \#2 & $\begin{array}{l}\text { ‘Chickenpox'[Mesh] OR varicella OR } \\
\text { 'Herpes Zoster'[Mesh] }\end{array}$ \\
\hline \#3 & \#1 OR \#2 \\
\hline \#4 & $\begin{array}{l}\text { epidemiology OR prevalence OR incidence } \\
\text { OR burden }\end{array}$ \\
\hline \#5 & $\begin{array}{l}\text { hospitalisation OR hospitalization OR } \\
\text { mortality OR 'case-fatality rate' }\end{array}$ \\
\hline \#6 & \#4 OR \#5 \\
\hline \#7 & \#3 AND \#6 \\
\hline$\# 8$ & 'Africa'[Mesh] \\
\hline$\# 9$ & 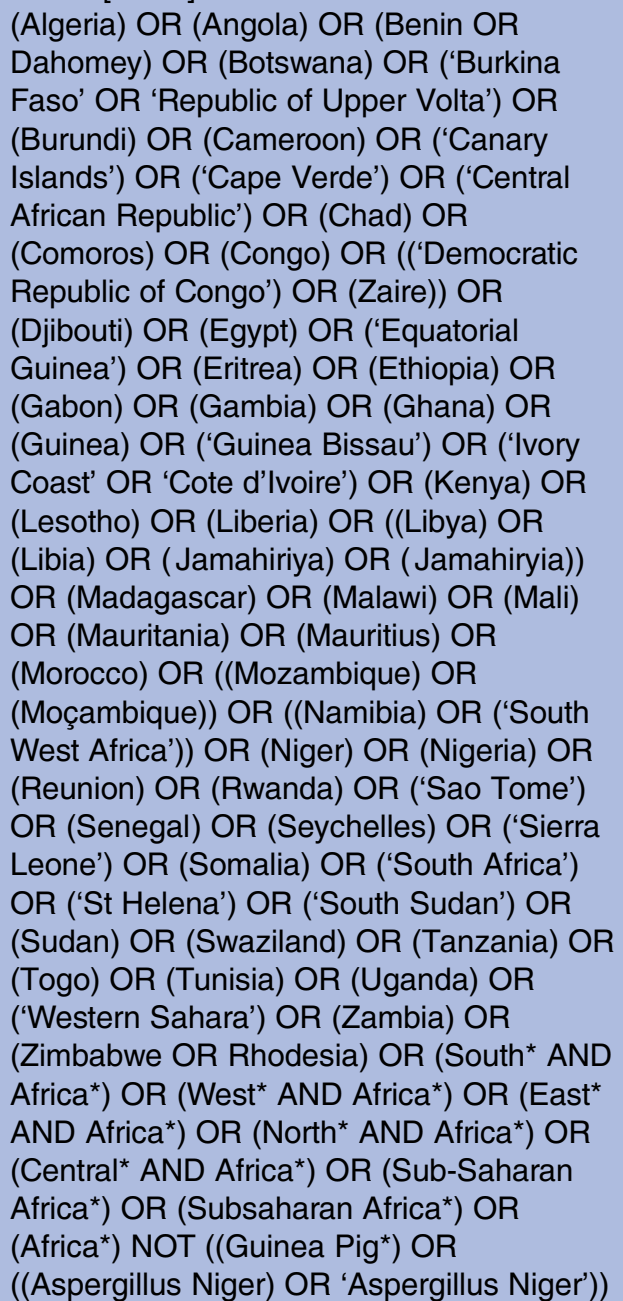 \\
\hline \#10 & \#8 OR \#9 \\
\hline \#11 & \#7 AND \#10 \\
\hline
\end{tabular}

study outcomes will be evaluated. The two authors will then independently read through the full text of all potentially eligible studies to assess if inclusion criteria are met. Discrepancies in the list of included studies between the two authors will be resolved through discussion and consensus, with the assistance of the last author (BMK). 
Table 2 Risk of bias and quality assessment for prevalence studies ${ }^{14}$

\begin{tabular}{|c|c|}
\hline Items & $\begin{array}{l}\text { Quality } \\
\text { score }\end{array}$ \\
\hline \multicolumn{2}{|l|}{ External validity } \\
\hline $\begin{array}{l}\text { 1. Was the study"s target population a close representation of the national population in relation to relevant } \\
\text { variables? }\end{array}$ & (1 point) \\
\hline 2. Was the sampling frame a true or close representation of the target population? & (1 point) \\
\hline 3. Was some form of random selection used to select the sample, or was a census undertaken? & (1 point) \\
\hline 4. Was the likelihood of non-response bias minimal? & (1 point) \\
\hline Total & (4 points) \\
\hline \multicolumn{2}{|l|}{ Internal validity } \\
\hline 1. Were data collected directly from the participants (as opposed to a proxy)? & (1 point) \\
\hline 2. Was an acceptable case definition used in the study? & (1 point) \\
\hline 3. Was the study instrument that measured the parameter of interest shown to have validity and reliability? & (1 point) \\
\hline 4. Was the same mode of data collection used for all participants? & (1 point) \\
\hline 5. Was the length of the shortest prevalence period for the parameter of interest appropriate? & (1 point) \\
\hline 6. Were the numerator(s) and denominator(s) for the parameter of interest appropriate? & (1 point) \\
\hline Total & (6 points) \\
\hline
\end{tabular}

\section{Data collection process}

Data will be independently extracted from the included studies by two reviewers, and recorded on a predesigned form. If need be, corresponding authors for the included studies will be contacted where the data are unclear. The following data will be extracted from the included studies:

- Study characteristics: year of the study publication, study design and objectives of the study

- Study population: country, community or healthcare facility based and the source of the denominator

- Case definition: laboratory methods and clinical case definitions

- Incidence or prevalence of VZV

- Mortality and hospitalisation rates associated with the VZV

- Prevalence of complications of VZV not requiring hospitalisation, for example, postherpetic neuralgia

- Characteristics of VZV cases: age, gender, HIV status, access to acyclovir or other antiviral treatment, vaccination status.

\section{Risk of bias assessment and strength of the cumulative} results for included studies

We will adapt the risk of bias and quality assessment tool developed by Hoy et $a l^{14}$ and modified by others for evaluating prevalence studies. ${ }^{15}$ This scoring tool, which will be used on all the included studies, examines the internal and external validity by taking into account study design, methodology and the presence of bias.

From the several quality assessment tools available, we chose to adapt the tool developed by Hoy et $a l^{14}$ for the following reasons: (1) it is specifically designed for prevalence studies; (2) it is an improved tool developed after a rigorous published process, including a review of the limitations of the existing tools; (3) detailed criteria to use the tool are provided, making it easier to use; (4) it has a high inter-rater agreement; and (5) it is robust in application and, for example, can be used alongside the latest tool developed by the Joanna Briggs Institute (JBI) and the Cochrane Collaboration. ${ }^{16}$

Two authors (HSH and LHA) will independently score the risk of bias using the tool and a $\kappa$ agreement will be calculated. This risk of bias and quality assessment tool is given in table 2. The strength of the cumulative evidence will be based on the average score obtained following the criteria described in table 2. An average score of 1,2, 3 and 4 points would imply the cumulative evidence is weak, average, strong and very strong, respectively.

This review will not include unpublished reports, and therefore is at risk of publication bias. As there is no standardised data collection for VZV across the continent, data from national health departments will not be used. The practical difficulties of collecting this set of data are a further reason for not collecting it, and this is a limitation of our study. Furthermore, if data are collected from health systems, they are automatically limited to medically attended cases and may miss milder cases or persons who have less access to healthcare services, which occurs commonly in Africa. We, therefore, acknowledge that our study is likely to underestimate the burden of the VZV-associated disease in Africa.

\section{Data synthesis}

The results from the included studies will be reported as incidence and/or prevalence of varicella or herpes zoster, as well as hospitalisation and death rates, using mean and SDs. A trend analysis will be conducted to investigate the trend of varicella or herpes zoster burden in Africa over time. R-programming software will be used to perform the statistical calculations.

If the studies included have a high heterogeneity (this will be examined by $\chi^{2}$ test of homogeneity and 
quantified using the $\mathrm{I}^{2}$ statistic) the findings will be presented in a narrative form that describes regions or countries with similar epidemiology of the disease.

\section{Subgroup analysis}

The findings from each country will be reported separately as part of a subgroup analysis. In addition, further subgroup analyses will be conducted, where sufficient data exist, based on whether the study was conducted in the community or in a healthcare centre, the income status of the countries as classified by the World Bank, national background HIV/AIDS prevalence rates, case definition criteria and geographical setting or population density (urban vs rural settings). ${ }^{17}$

\section{Sensitivity analysis}

For the meta-analysed data, a sensitivity analysis will be performed to determine if the exclusion of highly biased studies (based on the risk of bias assessment) will change the findings of the meta-analysis.

\section{Data management}

Data management will be the responsibility of the first author (HSH) in consultation with the last author (BMK). An electronic parent folder with the name of this study will be created. Subsequently, subfolders will be created that contain details of different tasks completed, such as all records retrieved, records included and excluded, risk of bias assessment results, analyses and full systematic review manuscript drafts. Two back-ups of the parent folder will be created, and stored on a memory stick as well as in a different computer.

\section{Reporting of the review}

The findings of this systematic review will be reported according to the Preferred Reporting Items for Systematic reviews and Meta-Analyses (PRISMA) guidelines, and will include a summary flow diagram on how articles were selected, as well as a list of excluded studies and the reasons for exclusion. Narrative reporting will be used to describe any qualitative data from the studies.

\section{DISCUSSION}

While there is a relatively large amount of epidemiological data on VZV in high income countries, there are only limited data from African countries. As there are major differences in the epidemiology of VZV-associated diseases between high, and low or middle income countries (LMIC's), data from high income countries cannot be extrapolated to LMIC's. A review from Latin America, for instance, found a much higher incidence of varicella compared with that in the USA, while a review from Asia found that a significant proportion of individuals are infected with varicella for the first time only after childhood. ${ }^{3} 18$ Our proposed systematic review will generate useful data on the burden of VZV in Africa, taking into account the high burden of HIV/AIDS. These results could be used as the first step towards developing vaccination control plans for varicella and zoster on the continent.

If the VZV-associated disease burden in Africa is found to be high, follow-up studies would be needed to determine the cost-effectiveness and feasibility of vaccination in this setting. It has been suggested that universal infant immunisation against varicella would not be possible in LMIC's, and that high-risk groups, such as immunocompromised individuals or healthcare workers, should be targeted for vaccination instead. ${ }^{1}$ But here again, more epidemiological data would be needed to inform such decisions. Furthermore, if the rates of VZV complications and/or hospitalisation were found to be high, it would provide strong evidence for wider accessibility of drugs such as acyclovir or those needed to treat post-herpetic neuralgia on the continent.

One of our study limitations is that only severe cases of VZV-associated cases are hospitalised or reported and in Africa, poor access to healthcare services is prevalent. Therefore, we are very likely to underestimate the burden of these diseases in our review. As such, even if our study finds the VZV-associated disease burden is low on the continent, we suggest that this review needs to be continuously updated. In addition, access to healthcare should improve over time and more data on VZV-associated cases will become available. Updating the review is also important as the epidemiology of many diseases, including varicella and zoster, will continue to change over time, as demographic changes occur and urbanisation increases. It is also important that the background epidemiology of vaccine-preventable diseases on the continent is known and updated regularly, as part of a general surveillance programme and for the review of vaccination policies.

\section{Author affiliations}

${ }^{1}$ Institute of Tropical Medicine and International Health, CharitéUniversitätsmedizin Berlin, Berlin, Germany

${ }^{2}$ Vaccines for Africa Initiative, Division of Medical Microbiology \& Institute of Infectious Disease and Molecular Medicine, University of Cape Town, Cape Town, South Africa

${ }^{3}$ Department of Biostatistics, School of Public Health, Harvard Medical School, Boston, Massachusetts, USA

${ }^{4}$ Department of Paediatrics \& Child Health, Groote Schuur Hospital, University of Cape Town, Cape Town, South Africa

Contributors HSH and GDH conceived the study. HSH developed the study protocol and will implement the systematic review under the supervision of BMK. JEC provided the statistical analysis plan of the study and will aid in the final data analysis. HSH and LHA will perform the study search, screening and extraction of data under the guidance of JEC, RM, GDH and BMK. HSH wrote the first draft of the protocol. All the authors gave input to the final draft of the protocol.

Funding The Vaccines For Africa Initiative (VACFA) will fund the costs associated with the dissemination of the results, including publications.

\section{Competing interests None declared.}

Provenance and peer review Not commissioned; externally peer reviewed.

Open Access This is an Open Access article distributed in accordance with the Creative Commons Attribution Non Commercial (CC BY-NC 4.0) license, which permits others to distribute, remix, adapt, build upon this work 
non-commercially, and license their derivative works on different terms, provided the original work is properly cited and the use is non-commercial. See: http://creativecommons.org/licenses/by-nc/4.0/

\section{REFERENCES}

1. Heininger U, Seward JF. Varicella. Lancet 2006;368:1365-76.

2. [No authors listed]. Varicella and herpes zoster vaccines: WHO position paper, 20 June 2014. Wkly Epidemiol Rec 2014;89:265-87. (cited 12 August 2015). http://www.who.int/wer/2014/wer8925/en/

3. Lee BW. Review of varicella zoster seroepidemiology in India and Southeast Asia. Trop Med Int Health 1998;3:886-90.

4. Arvin AM. Varicella-zoster virus. Clin Microbiol Rev 1996;9:361-81.

5. Thomas SL, Hall AJ. What does epidemiology tell us about risk factors for herpes zoster? Lancet Infect Dis 2004;4:26-33.

6. Gershon AA, Gershon MD, Breuer J, et al. Advances in the understanding of the pathogenesis and epidemiology of herpes zoster. J Clin Virol 2010;48(Suppl 1):S2-7.

7. Yawn BP, Gilden D. The global epidemiology of herpes zoster. Neurology 2013;81:928-30.

8. [No authors listed]. Varicella vaccines. WHO position paper. Wkly Epidemiol Rec 1998;73:241-8. (cited 8 September 2015). http:// www.who.int/docstore/wer/73 27 52.html

9. WHO. Global immunization vision and strategy. WHO. (cited 16 August 2015). http://www.who.int/immunization/givs/en/
10. Bendavid E, Ford N, Mills EJ. HIV and Africa's elderly: the problems and possibilities. AIDS 2012;26(Suppl 1):S85-91.

11. Hall V, Thomsen RW, Henriksen O, et al. Diabetes in sub Saharan Africa 1999-2011: epidemiology and public health implications. A systematic review. BMC Public Health 2011;11:564.

12. Varicella 2010 Case Definition. (cited 17 August 2015). http://wwwn. cdc.gov/nndss/conditions/varicella/case-definition/2010/

13. Centers for Disease Control and Prevention. Shingles (Herpes Zoster) diagnosis \& testing. http://www.cdc.gov/shingles/hcp/ diagnosis-testing.html

14. Hoy D, Brooks $P$, Woolf $A$, et al. Assessing risk of bias in prevalence studies: modification of an existing tool and evidence of interrater agreement. J Clin Epidemiol 2012;65:934-9.

15. Werfalli M, Musekiwa A, Engel ME, et al. The prevalence of type 2 diabetes mellitus among older people in Africa: a systematic review study protocol. BMJ Open 2014;4:e004747.

16. Munn Z, Moola S, Riitano D, et al. The development of a critical appraisal tool for use in systematic reviews addressing questions of prevalence. Int J Health Policy Manag 2014;3: 123-8

17. Country and Lending Groups Data. 2013 (cited 2 September 2015). http://data.worldbank.org/about/country-and-lending-groups

18. Bardach A, Cafferata ML, Klein K, et al. Incidence and use of resources for chickenpox and herpes zoster in Latin America and the Caribbean-a systematic review and meta-analysis. Pediatr Infect Dis J 2012;31:1263-8. 